\title{
Betaine biosynthesis in a heterologous expression system based on the B12 producer Pseudomonas denitrificans
}

\author{
Yuri Shkryl ${ }^{1, *}$, Anton Degtyarenko ${ }^{1}$, Valeria Grigorchuk ${ }^{1}$, Larisa Balabanova ${ }^{2,3}$, and \\ Lyudmila Tekutyeva ${ }^{3}$ \\ ${ }^{1}$ Federal Scientific Center of the East Asia Terrestrial Biodiversity, Far Eastern Branch of the Russian \\ Academy of Sciences, 690022 Vladivostok, Russia \\ ${ }^{2}$ G.B. Elyakov Pacific Institute of Bioorganic Chemistry, Far Eastern Branch of the Russian Academy \\ of Sciences, 690022 Vladivostok, Russia \\ ${ }^{3}$ Far Eastern Federal University, 690922 Vladivostok, Russia
}

\begin{abstract}
Glycine betaine is an important donor of methyl groups in various metabolic processes of the cell and acts as an osmoprotector when exposed to various abiotic stresses in pro- and eukaryotic organisms. Moreover, exogenous application of betaine activates the production of vitamin B12 in industrial strains-producers. In this work, we have developed a new technology for microbiological betaine synthesis that can be used in biotechnology to activate B12 biosynthesis during large-scale fermentation of Pseudomonas denitrificans.
\end{abstract}

\section{Introduction}

Vitamin B12 (cyanocobalamin): the vitamin is an antianemic factor. In the animal body, it plays a significant role in the process of hematopoiesis, the work of the red bone marrow and the biosynthesis of nucleic acids. Insufficient supply of vitamin B12 in pigs, poultry, and young ruminants causes malignant anemia (impoverished blood), accompanied by a sharp decrease in productivity, growth cessation, and complete exhaustion of the body due to low absorption of proteins and plant-based feed.

Known producers of cobalamin are prokaryotes and, above all, propionic and methaneforming bacteria, which also form this vitamin under natural conditions. High cobalamin titers (up to $200 \mathrm{mg} / \mathrm{l}$ ) were achieved for the optional anaerobe Propionibacterium shermanii, but the most common producer in the industry today is Pseudomonas denitrificans, which produces similar titers of the target product - B12 [1].

Such well-known inducers of vitamin synthesis as betaine, choline, and cobalt can make a significant contribution to optimizing the cultivation conditions of $P$. denitrificans as a $\mathrm{B} 12$ producer [2]. Usually, to increase B12 production, chemically pure betaine is added to the culture medium of the producer at a concentration of 5-7 g/l during 50-140 hours of fermentation [3]. However, the need for constant application of exogenous betaine leads to

\footnotetext{
* Corresponding author: yn80@mail.ru
} 
an increase in the cost of production of B12 [4;5]. As one of the alternative sources of betaine, its intracellular synthesis by a recombinant B12-producing bacterium can be used, for example, due to the heterologous expression of its biosynthesis genes from available substrates.

Despite de novo inability to synthesize betaine, bacteria are able to enzymatically convert choline to betaine in a two-step enzymatic reaction. At the same time, choline itself, transported to the cell through systems with high and low affinity - BetT and ProU, respectively [6], is first oxidized to glycine-betaine-aldehyde by the enzyme choline dehydrogenase (BetA). The second stage of oxidation, catalyzed by glycine-beta-aldehyde dehydrogenase (BetB), then converts glycine-beta-aldehyde to glycine betaine [7]. However, the availability of choline - a substrate for the synthesis of glycine betaineб is a limiting factor in the biosynthesis of this compound [8].

Recently, an alternative pathway for betaine biosynthesis has been described in which the available amicoacid, glycine, is used as a precursor [9]. A new pathway was discovered in the halotolerant cyanobacteria Aphanothece halophytica and we consists of a 3-stage sequential reaction of glycine methylation, which is carried out by a pair of $\mathrm{N}$ methyltransferase enzymes. One enzyme [A. halophytica glycinsarcosine methyltransferase $(A p G S M T)]$ catalyzes the methylation reaction of glycine to sarcosine and sarcosine to dimethylglycine, respectively, and the other [A. halophytica dimethylglycine methyltransferase $(A p D M T)]$ catalyzes the specific methylation of dimethylglycine to betaine [10].

\section{Materials and methods}

A DNA fragment containing the chimeric GSMT/DMT gene was obtained by polymerase chain reaction (PCR) using A. halophytica DNA as a matrix. ApGSMT-XbaI-dir and ApGSMT-linker-rev primers were used to obtain the ApGSMT gene, where ApGSMTXbaI-dir is a primer specific to the $\mathrm{N}$-terminal sequence of $A p G S M T$, including the site for XbaI restriction; ApGSMT-linker-rev is a reverse primer specific to the C-terminal sequence of $A p G S M T$, including the GGGGS linker sequence:

ApGSMT-XbaI-dir: 5'-TATCTAGAATGGCTATCAAAGAAAAAC-3'

ApGSMT-linker-rev: 5'-

CAGAGCCACCTCCGCCTGAACCGCCACCACCTGAATCTTTTTTCGCAACGTG-3'.

To obtain the ApDMT gene, primers ApDMT-linker-dir and ApDMT-HindIII-rev were used, where ApDMT-linker-dir is a primer specific to the N-terminal sequence of $A p D M T$, including the GGGGS linker sequence; ApDMT-HindIII-rev is a reverse primer specific to the C-terminal sequence of $A p D M T$, including the site for HindIII restrictase:

ApDMT-linker-dir: 5'CAGGCGGAGGTGGCTCTGGCGGTGGCGGATCGACTAAAGCAGACGCAGTCGC$3^{\prime}$

ApDMT-HindIII-rev: 5'-TTAAGCTTCTAGGGTTTGTGGAACTTG-3'.

After amplification, PCR products were mixed and repeated PCR was performed with ApGSMT-XbaI-dir and ApDMT-HindIII-rev primers.

The resulting fragment of the GSMT/DMT chimeric gene and pMF230 plasmid DNA were treated with $\mathrm{XbaI}$ and HindIII restrictases, then cross-linked using a ligase reaction. The reaction mixture was used to transform competent E. coli Rosetta (DE3) cells. E. coli cells were used to isolate pMF230-GSMT/DMT DNA (Fig. 1).

Recombinant $P$. denitrificans strain-producer was obtained by electroporation of $P$. denitrificans CCUG 1783 cells with recombinant pMF230-GSMT/DMT plasmid. Colonies of $P$. denitrificans were collected from the surface of LB-agar and cultured for a day in a liquid LB-medium. To determine the productivity of the strain $P$. denitrificans/pMF230- 
GSMT/DMT cells were incubated in a three-liter flask in a liquid LB-medium for a day at $25^{\circ} \mathrm{C}$ with intensive stirring $(200 \mathrm{rpm})$. The biomass was separated from the culture medium by centrifugation and washed with saline on filter paper. The suspension was disintegrated in a mixture of methanol/water $1 / 1(\mathrm{~V} / \mathrm{V})$. The resulting suspension was incubated at room temperature in an ultrasonic bath for 1 hour. After incubation, the suspension was centrifuged at $17000 \mathrm{~g}$ for 20 minutes. After that, the transparent supernatant was selected and transferred to new tubes for repeated centrifugation under the same conditions, followed by the selection of the supernatant for HPLC and MS analysis.

Identification of the obtained recombinant polypeptide is carried out according to characteristic mass spectrum peaks of glycine betaine: $[\mathrm{M}+\mathrm{H}]+=118.1$ and $[2 \mathrm{M}+\mathrm{H}]+=235$ using the device Bruker HCT Ultra core facilities in the CUS of Biotechnology and Genetic Engineering FSC of Biodiversity FEB RAS.

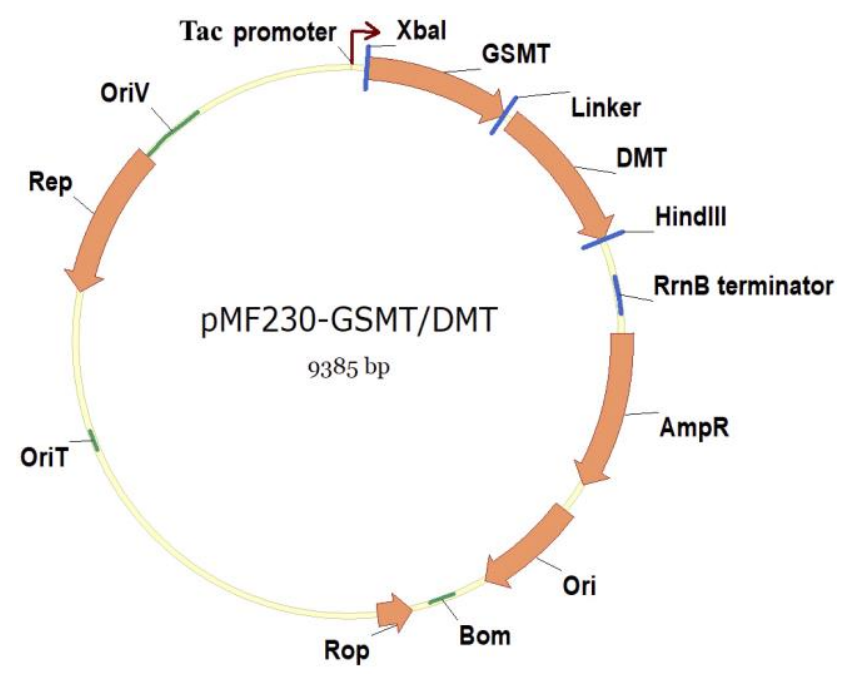

Fig. 1. Recombinant plasmid DNA pMF230-GSMT/DMT, carrying a chimeric sequence consisting of the coding part of the ApGSMT genes and fused together using a linker sequence.

\section{Results and discussion}

At the first stage of our work, we created a chimeric gene (GSMT/DMT) consisting of fused ApGSMT and ApDMT coding sequences connected by a flexible linker. The chimeric gene was placed in a plasmid pMF230 with a wide range of hosts [11], the expression of which is controlled by a strong synthetic tac promoter [12]. Previously, it was shown that the joint heterologous expression of these two enzymes in the cyanobacterium Synechococcus sp. PCC 7942 and Arabidopsis thaliana and Oryza sativa plants leads to the accumulation of a significant amount of betaine, the level of which significantly exceeded the indicators of transgenic cells expressing enzymes of the choline-dependent pathway [13; fourteen].

To test the assumption that the chimeric GSMT/DMT gene can induce betaine biosynthesis, the recombinant plamside pMF230-GSMT/DMT was transferred to $P$. denitrificans cells by electroporation. After the selection of transformed colonies, the recombinant strain was planted in a 11 storage culture. Like most prokaryotes, members of the Pseudomonas genus are not able to synthesize choline and betaine de novo [15], and exogenous choline transporters are ubiquitous in this group based on analysis of current genome sequences [16].

The HPLC-MS method was used to identify betaine in a recombinant strain of $P$. denitrificans. To identify the peak, the glycine betaine standard was used (Fig. 2), as well as 
by analyzing the decay products (daughter ions) (Fig. 3). Thus, we have shown that heterologous expression of the GSMT/DMT chimeric gene can provide a high level of betaine accumulation in the recombinant $P$. denitrificans strain. It is important to note that both in the original bacterium and in transgenic organisms carrying the GSMT and DMT enzymes, betaine synthesis is performed de novo and the addition of exogenous glycine did not significantly affect its production [17]. It has been shown that the accumulation of betaine in pro- and eukaryotic cells significantly increases their resistance to abiotic stresses [18].

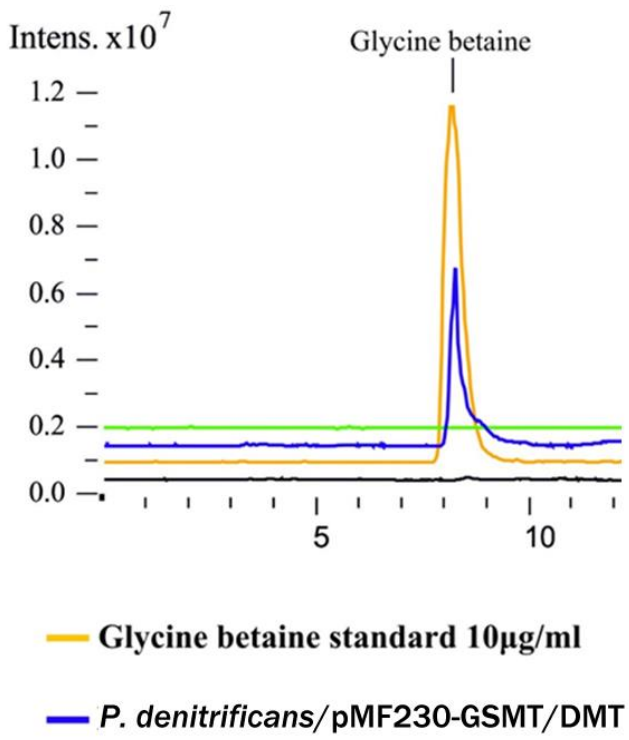

Fig. 2. Chromatography of the isolated ion; Intens - relative value of the ion current intensity.

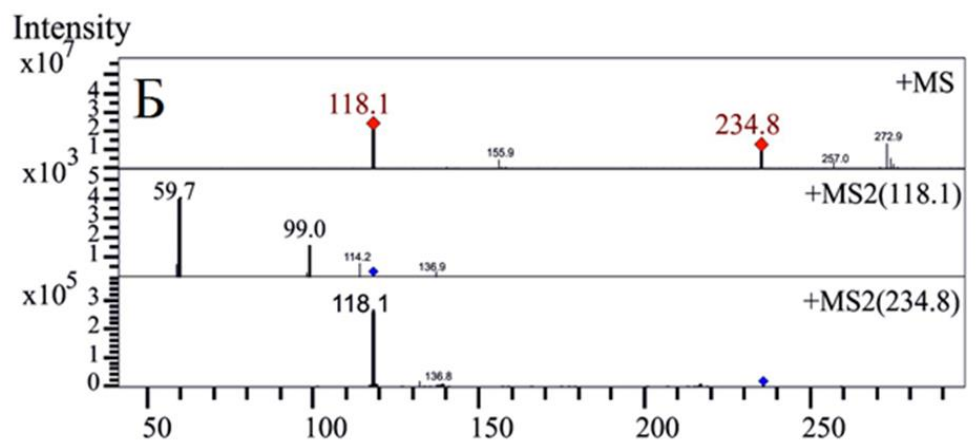

Fig. 3. MS and MS2 spectrum of ions with a molecular weight of 118 and 235.

\section{Conclusions}

Glycine betaine protects cells from negative abiotic stress factors by maintaining osmotic balance with the environment and stabilizing the quaternary structure of proteins. In addition, betaine is a supplier of methyl groups in general metabolism and activates the biosynthesis of an important anti-anemic vitamin - B12. However, the availability of a choline substrate for the synthesis of glycine betaine is a limiting factor in the production of this compound.Using heterologous expression of the GSMT/DMT chimeric gene in $P$. 
denitrificans cells, a high betaine content was achieved by de novo biosynthesis. This work shows for the first time a method for bioengineering betaine synthesis based on the use of an artificial protein with several catalytic functions. It is important to note that recombinant cells use the available amino acid glycine as a substrate for betaine biosynthesis in the developed technology, rather than molecules of choline that is deficient for bacteria. The described approach can be used in the biotechnological industry to increase the resistance of recombinant bacterial strains to adverse abiotic factors, as well as to activate the synthesis of valuable metabolites, such as cobalamin.

\section{Acknowledgements}

The work was performed within the framework of the R\&D of the Ministry of Industry and Trade of Russia No. 020-11-2018-1290.

\section{References}

1. J. Marie Sych, C. Lacroix, M.J.A. Stevens, Vitamin B12-physiology, production and application, In Industrial Biotechnology of Vitamins, Biopigments, and Antioxidants, (Wiley-VCH Verlag GmbH \& Co. KGaA; 2016)

2. Xia W, Peng WF, Chen W, Li KT., J Ind Microbiol Biotechnol 2015; 42:273-278

3. K.T. Li, D.H. Liu, Y.L. Li, J. Chu, Y.H. Wang, Y.P. Zhuang, S.L. Zhang, Bioresour Technol, 99, 8516-8520 (2008)

4. W. Xia, W.F. Peng, W Chen, K.T. Li. , J Ind Microbiol Biotechnol, 42, 273-278 (2015)

5. T. Lamark, O.B. Styrvold, A.R. Strøm, FEMS Microbiol Lett, 96, 149-154 (1992)

6. B. Landfald, A.R. Strøm, J Bacteriol, 165, 849-855 (1986)

7. S.D. McNeil, D. Rhodes, B.L. Russell, M.L. Nuccio, Y. Shachar-Hill, A.D. Hanson, Plant Physiol,124, 153-162 (2000)

8. R. Waditee, Y.Tanaka, K. Aoki, T. Hibino, H. Jikuya, J. Takano, T. Takabe, J Biol Chem, 278, 4932-4942 (2003)

9. D.E. Nivens, D.E. Ohman, J. Williams, M.J. Franklin, J Bacteriol, 183, 1047-1057, (2001)

10. H.A. de Boer, L.J. Comstock, M. Vasser, Proceedings of the National Academy of Sciences of the United States of America, 80(1), 21-25 (1983)

11. R. Waditee, M.N. Bhuiyan, V. Rai, et al., Proc Natl Acad Sci U S A, 102, 1318-1323 (2005)

12. X. Niu, F. Xiong, J. Liu, Y. Sui, Z. Zeng, B-R. Lu, Y. Liu. Environmental and Experimental Botany, 104, 16-25 (2014)

13. O. Geiger, I.M. López-Lara, C. Sohlenkamp, Biochim Biophys Acta, 1831, 503-513 (2013)

14. G.L. Winsor, D.K. Lam, L. Fleming, R. Lo, M.D. Whiteside, N.Y. Yu, R.E. Hancock, F.S. Brinkman. Nucleic Acids Res, 39, D596-600 (2011)

15. R. Waditee, M.N. Bhuiyan, V. Rai, et al. Proc Natl Acad Sci U S A, 102, 1318-1323 (2005)

16. D. Cleland, P. Krader, C. McCree, J. Tang, D. Emerson, J Microbiol Methods, 58, 31 38 (2004)

17. J. Giri, Plant Signal Behav, 6, 1746-1751 (2011) 